
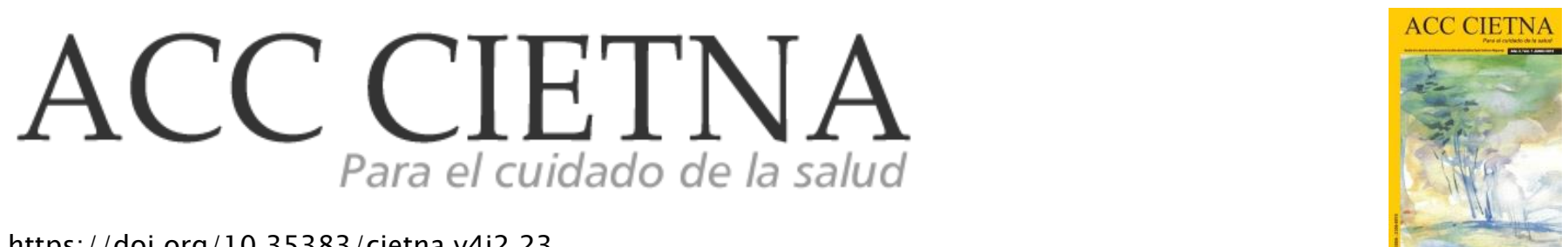

https://doi.org/10.35383/cietna.v4i2.23

\title{
Percepción de las enfermeras y las pacientes sobre el respeto a la intimidad durante el proceso hospitalario
}

\author{
Ramos Castro Roxana del Rosario ${ }^{1}$, Paredes Olano María Estela², Cervera Vallejos Mirtha Flor ${ }^{3}$
}

\begin{tabular}{l} 
INFORMACIÓN DEL ARTÍCULO RESUMEN \\
\hline Historia del artículo:
\end{tabular}

Recibido el 23 de mayo de 2016

Aceptado el 15 de setiembre de 2016

\section{Palabras claves:}

Percepción

Respeto

Intimidad

Enfermería

Hospitalización

Estudio de tipo cualitativo, que utilizó como abordaje metodológico estudio de caso, sus objetivos fueron caracterizar, analizar y comprender la percepción de enfermeras y pacientes sobre el respeto a la intimidad en el servicio de ginecología del Hospital Regional Docente las Mercedes de Chiclayo. La obtención de datos se aplicó la entrevista semiestructurada a profundidad y la observación no participante, estos datos se procesaron a través del análisis de contenido temático y de la técnica de triangulación. El estudio se desarrolló a la luz de los principios éticos de Ellio Sgreccia y de rigor científico por Guba y Linconl. Las categorías emergidas fueron: Apreciaciones de enfermeras y pacientes sobre el respeto a la intimidad, desposesión de la intimidad de la paciente mujer, condiciones desfavorables para ejercer el cuidado de la intimidad y reacciones de la mujer frente a la intimidad vulnerada. Los resultados demostraron que las enfermeras perciben la intimidad como una dimensión holística, pero se ve violentada por el gran número de pacientes, la inadecuada infraestructura, los deficientes recursos materiales, la intervención de otros profesionales, deficiente empatía, formación y resistencia al cambio. La mujer percibe la intimidad como el respeto a su cuerpo, enfocado a sus partes íntimas, asegurando sufrir

\footnotetext{
1 Licenciada en Enfermería. Residencia UCl - Hospital Cayetano Heredia - Lima, Perú. Email: roxana18_16@hotmail.com

2 Licenciada en Enfermería. Enfermera con trabajo independiente, Chiclayo, Perú. Email: stl_mepo@hotmail.com

${ }^{3}$ Doctora en enfermería. Docente de la Escuela de Enfermería de la Universidad Católica Santo Toribio de Mogrovejo,Chiclayo, Perú. Email: mcervera@usat.edu.pe
} 
situaciones de desposesión durante los cuidados, por falta de vocación y empatía, manifestando actitudes pasivas, sentimientos de vergüenza e incomodidad, tristeza y llanto, justificando dichas reacciones de pasividad por estar enferma, sentirse vulnerable y por miedo a que los profesionales tomen venganza, renieguen o se desquiten con ellas.

Perception of nurses and patients on respect for privacy during hospitalization process

ABSTRACT

\section{Keywords:}

Perception

Respect

Privacy

Nursing

Hospital
Qualitative study that used as a case study methodological approach, its objectives were to characterize, analyze and understand the perception of nurses and patients on respect for privacy in the service of gynecology at Mercy Regional Teaching Hospital of Chiclayo. The data collection semi-structured in-depth interview and non-participant observation were applied, these data were processed through thematic content analysis and triangulation technique. The study was conducted in the light of the ethical principles of Ellio Sgreccia and scientific rigor by Guba and Linconl. The emerged categories were: Appreciation of nurses and patients on respect for privacy, dispossession of the privacy of the patient women, unfavorable to exercise care privacy and reactions of women facing conditions privacy violated. The results showed that nurses perceived privacy as a holistic dimension, but is violated by the large number of patients, inadequate infrastructure, poor material resources, the involvement of other professionals, lacking empathy, training and resistance to change. The woman perceived privacy and respect your body, focused on her private parts, ensuring suffer situations of dispossession during care, lack of vocation and empathy, expressing passive attitudes, feelings of shame and embarrassment, sadness and sorrow, justifying such passive reactions by being sick, feeling vulnerable and afraid that professionals take revenge, or renege desquiten with them. 


\section{Introducción}

La intimidad representa lo más nuclear de la persona y debe ser protegida, pues es el mundo interior, el santuario de lo humano, un lugar donde solo entra la propia persona, porque allí radica sus vivencias personales, es decir, toda una analítica existencial cuyo perímetro gira en torno al ámbito nuclear del yo personal1,2,3. Por ello, la intimidad como dimensión exige una actitud de respeto por parte de la enfermera, pues las situaciones propias del cuidado develan aspectos, que normalmente quedan reservados para las relaciones especiales y limitadas de familiaridad, poniendo a la persona cuidada en condiciones que le fuerzan a compartir aspectos de esa intimidad suya con el cuidador ${ }^{4}$.

Ciertas situaciones propias del sistema sanitario también suponen amenazas para el respeto a la intimidad, como el número elevado de pacientes y acompañantes, situaciones de aglomeración, rapidez de la atención, la complejidad del diagnóstico, la intervención de muchos profesionales sanitarios en escaso tiempo y espacio o falta de áreas adecuadas para la privacidad, estos factores crean un contexto donde se transgrede la intimidad del paciente ${ }^{5}$, además el uso de alta tecnología diagnóstica y terapéutica, hace que la persona pierda su identidad de su sujeto, reparándolo solo como "objeto deteriorado", olvidando que es un ser doliente, que está dispuesto a mostrar solo aquello que quiere mostrar, aunque a veces se ve forzado a revelar sensaciones íntimas, independientemente a cómo el hecho afecte a su salud ${ }^{6}$.

Esta situación, no es ajena en el área de ginecología del Hospital Regional Docente Las Mercedes de Chiclayo, espacio donde la persona permanece hospitalizada y se ve expuesta a muchas circunstancias que vulneran su intimidad. Esta compleja realidad que se vive actualmente, llevó a las investigadoras a querer conocer ¿Cómo es la percepción de las enfermeras y las pacientes sobre el respeto a la intimidad durante el proceso hospitalario en el Hospital Regional Docente Las Mercedes?, con el objetivo de poder caracterizar, analizar y comprender esa percepción.

Esta investigación se justifica porque el respeto a la intimidad es un derecho inherente a la naturaleza de la persona, naturaleza que no puede reducirse solo a exterioridad o a un "cuerpo medicalizado"7, a su vez este respeto es un valor innato en la acción de cuidar. Por ello no puede cosificarse, ni puede perder sus rasgos personales e individuales, mucho menos prescindirse de sus sentimientos y valores para solo identificarse con sus rasgos externos, para ser tratado como: "el señor de la cama número tal", "la señora del legrado". Esta tendencia actual despersonaliza al ser humano, viola sus derechos como persona y como paciente. En este sentido, la intimidad debe ser cuidada, custodiada y protegida de manera holística por todos profesionales del campo sanitario, en especial la enfermera, por la inmediatez del cuidado durante las 24 horas del día, ejerciendo un rol de acompañamiento durante el proceso hospitalario.

\section{Metodología}

Estudio de tipo cualitativo con abordaje metodológico estudio de caso, abordaje que permitió comprender e interpretar a profundidad el respeto a la intimidad, intentando encontrar el sentido de los mismos a partir de los significados que las personas les conceden. Los sujetos de estudio fueron 6 enfermeras asistenciales, las cuales cumplieron los siguientes criterios de inclusión: ser enfermeras que voluntariamente accedieron a colaborar con la investigación previo consentimiento informado, con cinco años de 
experiencia en el cuidado de la persona hospitalizada, fueron excluidas las enfermeras que estaban reemplazando vacaciones o descansos médicos o realizando pasantías o estudiando la especialidad. Con relación a las pacientes, fueron 11 mujeres entre 18 a 50 años hospitalizadas y que cumplían con los siguientes criterios de inclusión: mujeres mayores de edad, en condición estable durante la entrevista y que acepten participar en la investigación voluntariamente previo consentimiento informado, así mismo, se excluyó del estudio a las mujeres que en su historia clínica tengan como antecedente algún problema psiquiátrico como: psicosis, esquizofrenia, trastornos obsesivos compulsivos.

Los datos se recolectaron mediante la entrevista semiestructurada 8,9 logrando conocer a profundidad la percepción de las enfermeras y las pacientes sobre el respeto a la intimidad y de esa manera saber cómo se está cuidando esa dimensión del ser humano, las preguntas se formularon partiendo del objetivo de estudio.

Una vez recolectados todos los datos, se realizó el procesamiento de la información a través del análisis temático ${ }^{10}$, permitiendo descomponer el corpus en unidades para proceder posteriormente a su agrupamiento en categorías siguiendo el criterio de analogía, es decir, considerando similitudes o semejanzas que existen entre éstas en función de criterios preestablecidos. Para triangular y corroborar si lo que decían las enfermeras era verdadero, se hizo las siguientes preguntas a las pacientes: ¿Qué entiende usted por respeto a su intimidad durante la hospitalización?, ¿Qué cuidados brindados por la enfermera aseguran el respeto de su intimidad?, las investigadoras permanecieron muy atentas a las respuestas y se hizo otras preguntas (repreguntas) para profundizar los aspectos relacionados con el objeto de estudio.

\section{Resultados, análisis y discusión}

CATEGORIA I: APRECIACIONES DE ENFERMERAS $Y$ PACIENTES SOBRE EL RESPETO A LA INTIMIDAD DURANTE LA HOSPITALIZACIÓN.

1.1 Intimidad dimensión holística con trato digno.

La enfermera por ser profesional, percibe la intimidad como una dimensión holística, su formación viene enfocada a valorar al enfermo como ser completo, por ello, ellas aluden la complejidad de esta dimensión por ser algo personal e intransferible de cada individuo, y con distintas acepciones, dependiendo de las situaciones y contexto en el que se entienda. Lo consideran como algo muy íntimo, que incluye aspectos físicos, psicológico, social, etc. Estas apreciaciones por parte de las enfermeras se declaran en los siguientes discursos:

"Es respetar a la persona como ser humano, en toda su dimensión, tanto físico, biológico, social, económico, cultural. A lo físico se refiere a no exponer su cuerpo a la vista de todos, también cuando nos confiesan algo nosotros tenemos que respetar esa intimidad" EG3

Manifiestan que la intimidad es respetar el ámbito físico, psicológico, social, económico, sin embargo cuando explican cada ámbito se enfocan en el cuerpo y la confidencialidad considerando estos más importantes. El cuerpo sirve para expresar la interioridad, el mismo cuerpo está en la intimidad, por eso uno se viste; por eso cuando se hace referencia pública e inesperada de uno la reacción habitual es el sonrojo, el pudor, el hombre no se viste sólo porque haga frío; sino porque su cuerpo forma parte de su intimidad. A pesar que el cuerpo exige un respeto, debemos tener presente que no se trata con "cuerpos" sino con seres corpóreos, que no es solo un "caso" sino una persona.

También tenemos que mencionar que en el servicio de Ginecología, la zona genital es la más 
expuesta, ya que la gran mayoría de patologías a tratar en este servicio necesitan de la exploración continua de los genitales, por ello las pacientes relaciona la intimidad aún más con sus partes íntimas y exigen protección al sentir esta parte de su cuerpo expuesta. Estas afirmaciones se aprecian en sus discursos:

"Que no esté expuesta a todo el mundo, que no vean mi cuerpo, por primera vez en mi vida me pasa, estoy internada, todos estuvieron ahí mirándome" MG2

"Que nadie más me vea, es un tabú, porque da vergüenza estar con harta gente y el doctor que nos examina, porque da vergüenza que vean nuestras partes'MG8

La intimidad física o corporal es la más afectada y reconocida en las pacientes. Esto supone que nadie puede acceder al cuerpo de otra persona sin su permiso, ninguna que esa persona no consienta puede tocarle, observarle o invadirle. Corresponderá, por tanto, al enfermo decidir a quién y a qué da acceso, pues sabe que su cuerpo no es un trozo de materia orgánica, por eso exige respeto y lo manifiesta en las entrevistas, pues corresponde a la parte visible de su propia intimidad.

Las enfermeras manifiestan en sus discursos que la intimidad es una dimensión holística que debe ser cuidada, por otro lado los discursos de las pacientes mujeres, aluden a que intimidad es respetar su cuerpo, no exponerlas físicamente ante miradas de extraños, especialmente no exponer sus partes íntimas. Si realizamos una comparación, las pacientes solo mencionan un ámbito de la intimidad, la corporal, quedando muy limitada su percepción acerca de la intimidad.

1.2 Derecho a la intimidad y privacidad del cuerpo.

El derecho a la intimidad es un derecho subjetivo, de defensa de una parte de nuestra vida que queremos mantener reservada, y de la que tenemos plena disposición. Lo contempla desde el punto vista legal la Ley $N^{\circ} 26842$, o ley General de Salud ${ }^{11}$, manifiesta en el art $15^{\circ}$ : Toda persona usuaria de los servicios de salud, tiene derecho: a) Al respeto de su personalidad, dignidad e intimidad, b) A exigir la reserva de la información relacionada con el acto médico y su historia clínica, con las excepciones que la ley establece. c) A no ser sometida sin su consentimiento, a exploración, tratamiento o exhibición con fines docentes; situaciones que engloban derechos que todo profesional de salud especialmente las enfermeras debe respetar y cumplir. Las enfermeras lo reconocen en los diferentes discursos como derecho que debe ser protegido y cuidado.

"Es proteger sus derechos y está en manos de nosotras que se respete, siempre y cuando la enfermera tenga la capacidad y solvencia para realizarlo, por ejemplo si bien el medico te dice ponle una sonda ya depende de ti si colocas un biombo o no, entonces los derechos de las personas hospitalizadas están en manos de nosotras y es nuestro deber respetar ese derecho" EG 1

En uno de los discursos de las enfermeras menciona la capacidad y solventura para respetar la intimidad como derecho, si bien es cierto, la intimidad se ve vulnerada por las diferentes percepciones e ideas de los profesionales, de la cultura del servicio, ya que todos no ven a la persona como un ser holístico, pero, reconoce que depende de cada profesional proteger esa intimidad, pues es sujeto de nuestro cuidado, aludiendo que la decisión es más personal. Este cuidado no está sujeto a una indicación médica, el proteger la intimidad, dependerá de la sensibilidad y empatía de la enfermera para darse cuenta de que la persona que cuida es un ser viviente y no un simple objeto manipulable.

Es necesario recalcar que resultara éticamente condenable todo hecho que violente de alguna forma este derecho, por ello debemos tener cuidado pues la interacción que se establece entre un paciente y el profesional de la salud que lo 
atiende, es una relación de proximidad y las situaciones que se presentan exigen develar aspectos de su intimidad que en la vida común quedarían reservados a las relaciones de familiaridad. Las pacientes también lo reconocen:

"Nadie puede venir pasar y verte si tú no lo deseas. Está estipulado ante la ley que uno tiene derecho a la privacidad y a no ser violentada... es un derecho al cuidado de tu cuerpo. No somos animales somos seres humanos'MG10

La persona reconoce su situación de vulnerabilidad de exposición de su intimidad corporal y lo manifiesta que lo hace pues está enfermo, está plenamente consciente de que en ocasiones debe mostrar su cuerpo desnudo para que le sea realizado un examen físico con el objeto de que se le haga un diagnóstico, y es aquí donde el personal sanitario debe buscar un punto medio de manera que no se viole la intimidad del paciente, no se afecte su pudor y se respete al enfermo para que no se sientan usados sino importantes para los demás ${ }^{12}$.

CATEGORÍA II: DESPOSESIÓN DE LA INTIMIDAD DURANTE LA HOSPITALIZACIÓN DE LA PACIENTE MUJER

2.1. Perdida de la autonomía y corporalidad descubierta.

En las cuestiones de enfermedad debemos considerar a la persona como un ser autónomo, capaz de tomar decisiones sobre su salud o la falta de esta. Por ello, todas las acciones no solo dependerán del criterio de los profesionales, sino también de la persona que enferma, porque es quién dirige su propia manera de afrontar la enfermedad y decide cómo quiere ser atendido ${ }^{13}$. Pero la realidad encontrada difiere del cuidado ideal, muchos de los relatos muestran exigencia del respeto de su autonomía ante la pérdida de la misma.
Ayer me levantaron y me dijo vamos al baño para que te laves tus partes, le dije que saliera, pero dijo no, acá no estás en tu casa, me lavé de la forma que ella quiso, me incomodó porque no fue como quise, hay personas que no respetan eso. MG6

Los internos rodean la cama, empiezan a tocarnos... el doctor dice, ella tiene esto, tiene lo otro, me parece que es una falta de respeto a nosotros, aunque sea por obligación o por nosotros, deben decir, miren ellos están acá, van hacerle algunas preguntas, van a estudiar, permítanos hacer esto, pedir permiso pero no lo hacen, los doctores son déspotas, creen que son mejores que uno. MG3

Los relatos demuestran diferentes situaciones de pérdida de autonomía, quizá para algunos profesionales enfocados en el paradigma categorización, no sea importante este ámbito de la intimidad, pero para nosotras como investigadoras si lo es, hemos mencionado que el ser humano es un ser viviente, que siente, piensa y que necesita tomar sus propias decisiones, quizá el proceso de enfermedad sea una limitante para su autonomía, pero como profesionales debemos velar por la salud de las personas minimizando la pérdida de autocontrol y fomentando la autonomía de los pacientes desde la ética del cuidar.

El consentimiento informado no es una hoja, no solo debe realizarse en procedimientos donde el riesgo de la persona es mayor, o el acto de no hacerlo genere implicancias legales, el consentimiento es un proceso continuo de comunicación e información en la relación profesional de salud - persona, y se fundamenta en el reconocimiento como sujeto moral racional con autonomía para gobernar su propia vida, su esencia no es otra cosa que considerar que las decisiones de salud, sean autónomas por la persona para ser moralmente aceptables 14,15,16. Así lo mencionan las enfermeras entrevistadas: 
El consentimiento es fundamental porque toda persona tiene que tener noción de lo que se le va a realizar o que es lo que tiene, tiene derecho desde un inicio a saber su diagnóstico, debemos hacer que todo el tratamiento que pueda recibir tenga su autorización, porque desde que hacemos el llenado del consentimiento informado estamos respetando la intimidad que pueda tener durante los procedimientos de enfermería. Tenemos que hablarle, explicarle, porque a veces el médico solo pasa y no le explica nada y tú tienes que explicar el porqué de ese medicamento, o de su enfermedad. EG 1

La enfermera reconoce el consentimiento como fundamental en su actuar, como derecho inherente de la persona, a saber su diagnóstico o tratamiento que recibirá desde el inicio del proceso hospitalario, como fundamental para respetar esa intimidad durante los procedimientos de enfermería, pero no concuerda con lo que refieren las mujeres hospitalizadas pues ellas afirman que no les piden permiso, ni mucho menos se les responde cuando preguntan sobre la acción o horario del medicamento. A la vez, la enfermera se enfoca en que el consentimiento informado es un formato, pues menciona "hacemos el llenado", y el consentimiento no es formato. Tan solo con firmar este formato no se puede asegurar en su totalidad el respeto a su autonomía, por ende a su intimidad.

La intimidad corporal se mantiene a recaudo con la ropa, por ello, el ser humano al estar expuesto, se siente desposeído. Sin su permiso de ese algo íntimo pasa a ser públicamente enseñado, entonces siente vergüenza, e incluso rabia. Sin embargo, en el sentirnos sin quererlo indebidamente "transparentes" ante los demás está operando ya ese segundo sentimiento que insinuábamos: el pudor, la inclinación a poner la intimidad a cubierto de miradas extrañas. El pudor es el gesto y la reacción espontánea de protección de lo íntimo que precede a la vergüenza y le da a ésta un sentido positivo de preservación. Por ello, cualquier intervención enfermera que refiera exposición y contacto físico, debe conocer el grado de pudor de la persona a la que atiende para actuar con mayor sensibilidad y no ofenderla. No es válido pensar que cuando la persona se encuentra bajo nuestros cuidados podemos hacer lo que nos parezca con su cuerpo 17, 18. Aquí algunas manifestación de como las enfermeras protegen la intimidad corporal.

En cada procedimiento se debe buscar la individualidad, el respeto al paciente, evitando no exponerla ante los familiares $u$ otros pacientes durante el baño de esponja. EG2

Protegerla en todo momento cuando le realicemos algún examen, llevarla al servicio de tópico para algún examen pues allí hay más intimidad. Intervenimos haciendo el requerimiento de ropa, cubriendo a la paciente, pues las pacientes después de algún procedimiento terminan húmedas, prestamos ropa pero nunca se les deja desnudas, algo le ponemos. EG3

La mujer hospitalizada, exige que, a la hora de la exploración, o la realización de algún procedimiento, no se vaya más allá de lo estrictamente imprescindible, esto es que no se le someta a exposiciones o contactos físicos innecesarios. También atenta contra la intimidad del enfermo que su cuerpo sea explorado o visto por más personas de las necesarias, ya se trate de espectadores o de personal médico como relatan las enfermeras entrevistas. Hacen mención el uso de biombos y sabanas para preservar esa intimidad corporal; a pesar de la escases de esos recursos básicos para proteger el cuerpo, ofrecen otras opciones como ponerse al lado del paciente, donde será expuesto el cuerpo de la persona, llevarla a otra sala continua "tópico" para que puedan realizarse allí el procedimiento.

Sin embargo hemos encontrado muchas afirmaciones que manifiestan una desposesión de la intimidad corporal. Así lo detallan los siguientes discursos de las pacientes. 
Durante la limpieza vaginal todas las mañanas, no protegen, les digo señorita yo no estoy sangrando porque no quiero que me hagan la limpieza y me desnuden, te ve la del costado, las de al frente $y$ así hacen con todas, a veces en los procedimientos han tratado de protegerme pero llegan los médicos y se perdía todo pues no me protegían, porque todos me están mirando, no me tapan. MG2

En los discursos se observa con claridad que no se respeta ámbito corporal de la intimidad, y existe una contradicción entre los discursos de las enfermeras, que nos hacen dudar de lo ideal, de lo real. Las enfermeras reconocieron que la intimidad es una dimensión holística que debe ser cuidada, enfatizando incluso el aspecto corporal de la intimidad, quizá solo reconocen teóricamente la intimidad como dimensión única y holística de la persona, pero no la protegen o respetan, por ello, las mujeres hospitalizadas en el servicio de ginecología sienten la necesidad de solicitar que respeten su cuerpo al apreciar que son miradas por varias personas durante la visita médica y especialmente la forma como fueron tratadas, así mismo reclaman que el respeto a su cuerpo sea una realidad, y no sólo afirmaciones teóricas, como se aprecia en los discursos.

\subsection{Intimidad emocional violentada vs} Intimidad espacial limitada.

La intimidad también tiene un ámbito psicológico y espacial, porque como hemos mencionado el ser humano no es solo cuerpo, el cual ocupa un espacio, sino también afecta lo emocional; se da una alteración de la imagen; una transformación de la apropiación corpórea, del sentido del humor y de la consideración de uno mismo.

En la enfermedad hay una ruptura de la integridad; el paciente oscila entre la dependencia y la independencia; su cuerpo enfermo pierde vigor y coraje para resolver problemas y enfrentar la enfermedad. Existe una guerra entre el optimismo y la lamentación que ponen al paciente inestable y de mal humor; sus estados de ánimo se tornan ambivalentes ${ }^{14}$; entonces tal vez su única fortaleza estaría en permitir que otros lo cuiden, le ayuden, pero para que la persona acepte esa ayuda debemos acercarnos cuidadosamente, conociendo su cultura, su experiencia de salud, sus características propias, su grado de pudor, para actuar con máxima sensibilidad para no ofenderla con nuestra actuación.

Si la enfermera no se acerca, no expresa presencia cuidadora, y las mujeres perciben una comunicación no verbal nada adecuada con ellas, su comportamiento será negativo hacia los profesionales que la atienden como las mismas mujeres lo expresan:

Ese día que me han hecho el legrado el doctor me dijo ya pues ayúdame, tienes que cooperar, cállate, estás haciendo mucho ruido, yo recién te estoy empezando el procedimiento, como serás cuando estas con tu pareja, peor todavía me sentí mal, entonces creo eso es algo bien incómodo, yo creo que el cómo doctor me hubiera dado más aliento. MG4

Entre los actos de violencia a la intimidad psicológica se pueden mencionar los abusos emocionales, que incluyen cualquier forma de aflicción espiritual grave que sea generada por humillaciones, amenazas, hostigamientos u otras actitudes intimidantes, gritos, retos, etc. También la "falta de atención" o el trato frío, indiferente, impersonal y carente de empatía, o cuando los pacientes no son informados adecuadamente acerca de su dolencia y el tratamiento que van a recibir, o cuando no son escuchados o no reciben una respuesta cuando manifiestan su disconformidad con el tratamiento recibido como mencionan en los discursos expuestos.

Por otro lado, es necesario comprender que dadas las circunstancias de enfermedad, las mujeres están hospitalizadas en un servicio, ocupando un área geográfica. La intimidad espacial vendría a ser entonces, el territorio o espacio propio del 
paciente, es su cama o su cuarto: tanto el profesional de salud como los visitantes deben respetar este espacio, que es parte de su privacidad, procurando salvaguardar su intimidad, evitando comentarios inoportunos, o relacionados con la institución, con otros pacientes o con problemas familiares que no puede resolver en su lecho de enfermo.

Cuando nos limpian lo único que cierran es esa puerta de ahí, todo es al aire libre, los que pasan por ahí nos ven, los doctores, internos, tu como persona que no estas acostumbrada que todos te vean causa vergüenza, pero ellas son las enfermeras no podemos hacer nada, una persona se quejó la vez pasada y la enfermera le dijo pero si todas somos mujeres, además ellos son doctores, las ven, de que se avergüenzan. MG3

En caso de este hospital su infraestructura es un factor externo determinante para vulnera la intimidad territorial pues todos los pacientes se encuentran en una sala común a menos de un metro de distancia sin biombos que los separe. Entonces durante la exploración física del médico o la enfermera, o la realización de algún otro procedimiento la persona es expuesta no solo ante ellos, sino también a las miradas extrañas de alrededor de la habitación, es decir, de los demás pacientes hospitalizados y sus familiares presentes.

\subsection{Confidencialidad expuesta y trato} deshumanizado.

Las enfermeras reconocen que la confidencia se vulnera a través de la difusión de lo confiado en los siguientes discursos:

Se vulnera la intimidad con los diagnósticos, el personal debe ser cuidadoso al momento de decir el diagnostico, incluso no porque es su familiar tenemos el derecho de decir el diagnostico, no todas las personas desean eso, hay quienes ocultan su diagnóstico a ciertos familiares y debemos respetar eso. EG 1

El secreto profesional es una forma de secreto confiado o pactado, es decir, al asumir una profesión o al entablar una relación con un paciente, se establece un acuerdo tácito, por la cual la información que el paciente brinda, debe ser guardada bajo secreto, secreto que consiste en el compromiso de no divulgar aquello que es conocido mediante el desempeño del profesional, pues este compromiso fue adquirido al asumir la profesión ante la sociedad. Este secreto incluye tanto la naturaleza de la enfermedad como todas las circunstancias relacionadas, además de todas aquellas que, de ser reveladas, puedan ocasionar algún tipo de perjuicio al paciente 0 sus relacionados, incluyendo lo que sin haber sido explícitamente confiado al profesional haya sido conocido por éste en el ejercicio de sus funciones. Si bien es cierto que el paciente es una persona y como tal un ser libre que toma la decisión de consultar, al entregar información sensible desequilibra el poder e influencia que el profesional tiene sobre la persona, con el consecuente, pone el riesgo su autonomía y libertad ${ }^{19,20}$.

Las pacientes también reconocen situaciones donde se vulnera la confidencia:

Cuando pasan visita todas escuchan lo que tengo porque el medico viene con sus estudiantes, y se ponen a conversar, me preguntan, ni me explican nada, ellos hablan y hablan, las del costado escuchan todo, yo no digo nada porque ni entiendo, además si pregunto a veces se molestan o me responde mal. MG7

La persona informa de algunos hechos objetivos, algunos incluso observables por terceros, también comparte vivencias particulares de su ser íntimo, por ello requiere generar condiciones para una comunicación personal, ya que la confidencia y el desprendimiento de lo más íntimo de la persona se da durante la entrevista. La entrevista es un proceso de comunicación oportuna, generadora de confianza, pero este proceso debe desarrollarse en privacidad, en un ambiente que inspire comodidad, donde la persona pueda sentirse libre, 
sin presiones o miedo a que otros escuchen cuando expresa su sufrimiento. Esta situación no se da en el H.R.D.L.M. ya que el ambiente donde se realiza la entrevista, es una sala común, con 14 camas separadas en menos de un metro de distancia, sin biombos que los separe, es casi imposible evitar que los demás pacientes hospitalizados no escuchen las confidencias de la persona entrevista.

La humanización de los cuidados hace referencia a personas en interacción, que saben clarificar sus necesidades, reconocen sus sentimientos y se enfocan positivamente. Para ello, se requiere formar una enfermera con empatía, capaz de otorgar un cuidado familiar, significativo $y$ gratificante. Cuidar en enfermería implica el conocimiento de la persona a quien se brindará cuidado, conocer sus actitudes, aptitudes, intereses y motivaciones, además de los conocimientos que posee la enfermera, requiere manifestarse como persona: única, auténtica, capaz de generar confianza, serenidad, seguridad y apoyo efectivo ${ }^{21}$.

Pero las mujeres hospitalizadas mencionan que existe un trato deshumanizado como lo detallan en los siguientes discursos:

No te llaman por tu nombre, te dicen "la cama número once", "la señora del legrado", justo acaba de pasar la enfermera y ni te saluda. Te tratan como animal, te desnudan, te lavan, te dicen señora siéntese o cuéstese, nosotras queremos un mejor trato, no gritando. MG 8

Cuando viene hacer investigaciones los doctores y sus alumnos, te levantan la bata así no más, te revisan delante de todos, es una falta de respeto como seres humanos tenemos sentimientos. MG4

Es elemental llamar a la persona por su nombre y no por la patología que presenta, mantener un contacto a través de todos los sentidos con el paciente, nos mantendrá pendientes de los cambios que presente durante la hospitalización. Esta despersonalización creciente, en la que la persona ha pasado a un lamentable segundo plano ya sea por el aumento de la demanda asistencial o tecnificación de la atención de salud que otorgan los hospitales e instituciones como el HRDLM, donde la actitud humanizante que debe caracterizar la relación entre prestadores y usuarios se ha ido deteriorando, ha reducido la visión integral del paciente como ser biopsicosocial, adjudicándose el protagonismo a la enfermedad, por ello utilizamos la enfermedad para identificar o llamar a la persona.

En este punto, Gómez citando a Henderson ${ }^{14}$ señala que existen otros signos o situaciones en que las relaciones con el equipo de salud dejan de manifiesto el protagonismo de la enfermedad y en las que se exponen claramente a los pacientes/usuarios a un segundo plano, tales como el tutear a los adultos, dirigirse hacia otra persona sin identificarse previamente, hacer comentarios en presencia de otro paciente, realizar procedimientos sin el consentimiento, trasladar a los pacientes de sala sin explicación alguna.

CATEGORÍA III: CONDICIONES DESFAVORABLES PARA EJERCER EL CUIDADO DE LA INTIMIDAD.

3.1. Inadecuada valoración de la persona hospitalizada: escasa formación, deficiente empatía y resistencia al cambio.

Existen factores que contribuyen a una inadecuada valoración de la persona hospitalizada, una de ellas es la escasa formación, lo cual dificulta la relación enfermera - paciente durante el cuidado, así mismo la deficiente empatía y resistencia al cambio contribuyen a que no se visualice a la paciente como un ser unitotal, sino como un ser biológico que se puede reparar y coaccionar.

Para efectuar un trabajo con eficiencia, eficacia y efectividad requiere, además, de un pensamiento científico, un conocimiento actualizado y científicamente estructurado y del ejercicio de una 
práctica de enfermería sobre bases científicas 22. Para lograr lo descrito anteriormente, el personal debe realizar una capacitación constante $y$ permanente, ya que como se evidencia en los discursos, las enfermeras hacen referencia que la falta de compromiso de los gerentes y la poca iniciativa para capacitarse ha generado que se vulnere la intimidad de las mujeres hospitalizadas.

Esto sucede porque no hay la capacitación debida, los gerentes no se preocupan por capacitarte y el personal no está permanente capacitándose lo que hace que olvidemos todos estos aspectos del paciente, capacitación se refiere por ejemplo a la parte psicológica, temas relacionados a la intimidad del paciente, que lo puede dar muy bien un psicólogo, como también otros profesionales de enfermería y también leer, muchas veces ya no se lee, todo eso implica que se vulnera la intimidad del paciente. EG5

En este sentido para las enfermeras del servicio de ginecología, la deficiente capacitación es una condición desfavorable importantísima que se debe tener en cuenta para respetar la dimensión intimidad. Para Guevara23 la enfermera como integrante del equipo de salud tiene una gran responsabilidad: cuidar a las personas con compromiso profesional y ético, esto implica, velar por la dignidad y respeto que merece el ser humano; ya que éste es un ser complejo y digno por naturaleza, para ello debe capacitarse permanentemente sobre estos temas tal y como lo manifiestan en los siguientes discursos.

La capacitación es el mejor conocimiento para nosotras, para que se estimule, para que vuelva a recordar y ser mejor, también por parte de la jefatura estar pendiente de esas cosas ósea ver lo que falta, para que se pueda respetar la parte física del paciente y psicológica. EG5

El respeto de la intimidad debe estar en la formación de los profesionales de la salud, en todas las carreras deberían llevar un curso o algo sobre el respeto a la intimidad porque es parte del cuidado ético, es importante porque si no formamos a los alumnos, en el futuro vamos a seguir con esas conductas o peor, es necesario que se concienticen sobre que es el cuidado humano. EG3

Según los discursos mencionados, las enfermeras consideran que todos los profesionales de la salud se deben capacitar, e incluso el personal de mantenimiento, porque el respeto de esta dimensión es un tema que compete a todos los que trabajan en el ámbito hospitalario, así mismo mencionan que en todas las carreras de salud se debería llevar un curso sobre respeto de la intimidad de la persona hospitalizada, para que en el futuro no se vulnere esta dimensión. Para el ello, el profesional debe estar comprometido personalmente y querer crecer, pues nadie educa a nadie, principalmente si éste no lo desea, ni nadie se educa a sí mismo; los hombres se educan entre sí, mediatizados por el mundo. Así como las enfermeras reconocen que una deficiente capacitación genera que se vulnere la intimidad, también reconocen que actualmente no practican la empatía, así como lo expresan en los siguientes discursos:

Creo que nos está faltando ponernos en el lugar de la persona, no manejamos bien los procesos para colocar en posición ginecológica, para brindar información, para cubrir y solo exponer la parte en donde el medico realiza el procedimiento. EG3

La empatía entendida como un sentimiento de participación afectiva, de un sujeto en una realidad ajena, ponerse en el lugar del otro. Durante el ejercicio de la práctica asistencial, surgen relaciones y comportamientos entre profesional y paciente. En ese sentido, el respeto de la intimidad exige la práctica de esa habilidad social tan importante, para generar condiciones de intimidad, de privacidad, que deben ser garantizadas, en el caso del cuidado, por las enfermeras, pues deben ser conscientes de que la intimidad que se le abre, ha de ser tratada de manera explícita, con sensibilidad y respeto, pues son las personas las que le otorgan un valor y el 
profesional quien ha de conocer y calibrar el mismo, para que la relación entre cuidador y ser cuidado encuentre armonía.

Si bien con el paso de los años la disciplina ha ido adquiriendo un cuerpo de conocimiento más amplio, éste fenómeno descrito de alguna manera también ha influido en la deshumanización de la atención brindada. Como lo explica Rodríguez ${ }^{24}$ citando a Vargas Tolosa, en muchas de las instancias ocurre "Una despersonalización (entendiendo esta como una falta de sensibilidad de los profesionales hacia la personan que cuidan), visualizando al paciente como sujeto de conocimiento, estableciendo que la experiencia vivida en relación a él, se convierta en objeto, descontextualizándola y vaciándola de subjetividad y sentido", así lo expresan los discursos dado por las enfermeras:

El cuidado se ha despersonalizado porque el médico es muy suelto a veces dice "la trece pásala a tópico", "pasa el cáncer", ahí se vulnera la intimidad, nosotras debemos de decirlo porque ellos no lo hacen, raro es el médico que Ilama por su nombre al paciente, están acostumbrados, pero nosotras no. EG1

El profesional se vuelve frio, por la rutina, porque se mecaniza, se cansa, algunas personas no todas, pero si la gran mayoría, si hay colegas que tratan bien y llegan al paciente, a veces tu tratas bien como enfermera, pero viene el médico y no lo hace, lo ideal es trabajar en conjunto para que el paciente se sienta bien durante su hospitalización. EG2

Como se observa en los discursos, las enfermeras coinciden en que hay una pérdida de la concepción de persona y se restringe la visión del individuo a un ente meramente biológico sobre el cual se aplican una serie de prácticas que lo convierten en objeto de exploración y que atentan contra su condición de ser humano sensible y pensante. Cabe mencionar que el mismo profesional ha contribuido a desvirtuar la esencia del cuidado enfocado en relaciones humanas y lo ha convertido en un espacio donde se da una atención forzada y despersonalizada.

Es importante indicar según los discursos, que el médico es el principal sujeto que vulnera la intimidad, ya que según los relatos, es muy común llamar a la mujer hospitalizada por el número de la cama en la que se encuentra, o en el peor de los casos, por el nombre de la patología que estén presentando. Esta situación atenta contra la naturaleza integral de la persona desde su complejidad e integralidad y la deshumaniza a partir de esa concepción biologista que la determina a partir de una parte de su cuerpo que no se encuentra bien. Así mismo las enfermeras tratan de respetar y hacer recordar al médico que debe llamar por el nombre a las pacientes sin embargo encuentra obstáculos como los que se mencionan en los siguientes discursos:

Hacer el cambio cuesta, porque a veces tú lo haces pero el resto de tus compañeras no, y ese es otro obstáculo porque entre las mismas colegas dicen allí se va la mala, no ella es buena, y si tú quieres hacer un cambio no lo puedes hacer porque el servicio ya tiene reglas. EG2

Es difícil cambiar la mentalidad, hay mucha resistencia al cambio, tú no puedes cambiar a las personas así por así, porque ya están formadas y es muy difícil pero sin embargo no es imposible. EG5

Las enfermeras mencionan que para que se respete la intimidad de la mujer debe haber cambios, sin embargo se encuentran con diversos obstáculos, uno de ellos es la resistencia al cambio, ya sea por la rutina o por los años de experiencia, otras lo atribuyen que así las formaron y es difícil cambiar su mentalidad. La resistencia al cambio es un fenómeno que se da tanto a nivel personal como a nivel organizacional. Esto se puede deber a un rasgo de personalidad llamado "apertura mental" (las que puntúan más en este rasgo son más proclives a aceptar situaciones nuevas) o al simple hábito (una persona que este habituada a cambiar de comportamientos o 
situaciones tendrá que realizar menos esfuerzo que otra que no). El cambio produce ansiedad ante una situación incierta; la persona percibe su sentido de seguridad y prefiere no salir de su status 25 .

Así mismo mencionan que para cuidar la dimensión intimidad, cada enfermera debe tener ética profesional y la nueva generación de estudiantes debe poner en práctica los nuevos conocimientos adquiridos y que vienen evolucionando constantemente, así como lo detallan en los siguientes discursos:

Depende de la nueva generación de enfermería para que eso cambie, nosotros somos enfermeras de muchos años, tenemos toda la práctica, pero ustedes los conocimientos que han ido evolucionando, o todo lo contrario actuaran como unas máquinas, ya no se acercaran a las pacientes quizá por computadoras o teléfonos, pero enfermería quiere decir acercamiento, una atención personalizada, humana. EG2

Las enfermeras son las responsables de garantizar el ejercicio del derecho a la intimidad de una manera más global, tanto por su permanecía y continuidad a lado del paciente en su recorrido por el sistema, ejerciendo un rol de acompañamiento, como por su propio ámbito disciplinar asentada en el ejercicio del cuidado, mediante su rol de garante. Al respecto la investigación la ética y moral de la enfermería citando a Colliére ${ }^{26}$ : menciona "Desempeñando el rol de enfermera del que está investida, la enfermera experimenta en el curso de su formación y durante el ejercicio profesional la influencia de corrientes predominantes que contribuyen a orientar de forma determinante los imperativos de su rol".

El respeto a la intimidad es una necesidad sentida por las mujeres del servicio de ginecología ya que ellas ven la hospitalización como una situación nueva, impredecible, despersonalizadora $y$, en muchas ocasiones, estresante, con deterioro importante de la intimidad, la confidencialidad y de la misma dignidad. Así como se menciona en los siguientes discursos:

Veo que las enfermeras no hacen nada por preservar la intimidad, creo que con el tiempo han perdido el sentido de vocación, consideración y conforme ganan experiencia se vuelven menos humanos lamentablemente. Hay que ser empáticos, todo vamos a caer enfermos y vamos a estar hospitalizados y hay que saber tratar, ponerse en el lugar cuando el paciente está con dolor. MG 10

Creo que deben mejorar las enfermeras, ser más solidarias, amables, sencillas, porque hay una que otra que son déspotas, te tratan mal, a veces no solamente te tratan mal a ti como paciente, tratan mal a las personas que están afuera, a tus familiares. MG3

A diferencia de las enfermeras, las pacientes aluden que el irrespeto de la intimidad es por falta de vocación, empatía, solidaridad y amabilidad. La vocación en enfermería refleja la personalidad de la profesión en cada uno de quienes la ejercen; viene inmersa en los valores y principios de cada uno; es un reflejo del cuidado que demuestra el profesional de enfermería. Al respecto Gonzales Burgos citando a Florencia Nightingale 27 menciona "una enfermera debe ser una persona en la que se pueda confiar, en otras palabras, capaz de ser enfermera de confianza... no puede ser ligera, habladora; nunca debe contestar preguntas sobre su paciente; debe ser estrictamente moderada y honesta".

Para las pacientes, el contar con una mano solidaria, empática y con agradable relación humana, de respeto y cuidado personalizado, es vital y papel de la enfermería, profesión que se debe ejercer con espíritu de servicio, buscando la excelencia en el cuidado y favoreciendo así la calidad en la atención.

3.2.- Inadecuada Infraestructura y recursos materiales.

La estructura física y los recursos materiales de los que dispone el hospital generan condiciones que 
en ocasiones son poco favorables para ejercer el respeto de la intimidad. En este sentido se cuestiona como debe ser la estructura física, cuantas habitaciones debe poseer un servicio, cuantos pacientes deben estar en cada servicio y que es mejor estar en una sala común o en una habitación individual, para estar en condiciones de respetar la intimidad de la mujer hospitalizada6. Algunos relatos mencionados por las enfermeras demuestran cómo es la estructura física del servicio de ginecología y en qué medida perjudica la atención de la paciente.

La infraestructura no ayuda mucho, el tener una sala común con una infraestructura antigua es inadecuado para cuidar la intimidad, ya que se realiza un examen en el ambiente propio del servicio, donde a veces la premura del tiempo, el estado del paciente, no nos permite proteger adecuadamente, a veces se les coloca en una posición ginecológica cubriendo la parte superior por la premura, por la emergencia o porque estamos dejando de lado ese cuidado que la enfermera debe brindar a la persona. EG3

El personal de enfermería admite que la estructura física del servicio, dificulta el cuidado de la intimidad, en el área de ginecología existe una sala común, en la cual hay 14 pacientes y la distancia entre las camas donde se acuestan las mujeres es menos de 1 metro, así mismo cada espacio no está separado por cortinas o biombos, más aun, en cada cama no figura el nombre de la persona lo que vulnera la individualidad. Esta situación se puede definir como déficit estructural de la protección de la intimidad corporal, dado que todavía existen hospitales con habitaciones compartidas por más de seis enfermos, con aseos en los pasillos, personal que entra a la habitación sin llamar a las puertas, excesivo número de personas que hacen visitas en las habitaciones sin el consentimiento del interesado y un largo etcétera.
Así como es imprescindible una adecuada estructura física, es también de suma importancia contar con recursos materiales que aseguren el respeto de esta dimensión. En el servicio de ginecología se pudo evidenciar la falta de materiales para el cuidado de la intimidad y esto se constató mediante la observación y los discursos emitidos por las mismas enfermeras y pacientes, así como se observa el los relatos:

Uno de los obstáculos es por ejemplo, la falta de materiales, el ambiente mismo, el ambiente es imposible, yo no lo puedo cambiar, el ambiente ya está hecho, pero sin embargo, puedo cerrar la puerta, puedo poner un biombo, aunque a este servicio falta biombos, pues solo tenemos dos, debemos coordinar con la jefatura para que mejore estas cosas. EG5

Debe haber biombos, pero aquí solo hay el que está en la puerta, todas estamos enfermas, como nos van a proteger si todas estamos iguales, vamos a pasar por lo mismo. MG1

Para las enfermeras y las pacientes los recursos materiales constituyen medios físicos y concretos que ayudan a conseguir un objetivo que es el cuidado de la intimidad. Las mujeres piden que se les cubra durante los procedimientos con biombos o cortinas, sin embargo son conscientes que el hospital no cuenta con estos recursos, por lo que aceptan que no se cubra porque aluden que están enfermas, que todas pasaran por lo mismo, y manifiestan como "pues nos van a proteger" .Para las enfermeras la importancia de lo percibido como "falta de materiales y no tenemos ropa" responde a que la enfermera jefe es la encargada de la provisión de materiales en el servicio que lidera, sus funciones son planificar, controlar, supervisar y regular las entradas, salidas y existencias de materiales y equipos; así mismo, vigilar el uso racional de los mismos. Estas restricciones que pueden tener significancia técnica, son percibidas como un "control" desmedido, lo cual aunado a las restricciones institucionales, conlleva a la 
insatisfacción del personal y genera que no se realice un adecuado cuidado de la intimidad.

\section{CATEGORÍA IV: REACCIONES DE LA MUJER FRENTE} A LA INTIMIDAD VULNERADA.

4.1. Reaccionando con actitud pasiva, llanto y tristeza.

Burgos $\mathrm{M}^{28}$ citando Rocha menciona "el adoptar una actitud pasiva frente a las conductas violentas puede relacionarse con la relación asimétrica y jerárquica que se establece entre el personal de salud y los pacientes en los hospitales públicos, en la que los pacientes en una situación de dependencia en mayor o menor grado toleran las diversas conductas y manifestaciones que surgen durante la hospitalización. En los siguientes discursos manifestados por las pacientes se evidencia reacciones de pasividad:

Nada porque cuando uno está vulnerable es cuando pasa eso, vulnerable es cuando estamos enfermos, porque a una persona sana tu no le vas hacer eso, se aprovechan de ti, toda la vida va hacer igual, sé que nunca van a respetar porque te ven que estas débil. MG6

Yo no digo nada, porque si uno le dice algo a una enfermera, se pone a renegar, a decir, todas somos mujeres, ellos son doctores, saben, ya las conocen. MG3

Como se observa en los discursos, las mujeres no hacen nada y aceptan con resignación el irrespeto de su intimidad, muchas veces porque se sienten vulnerables, sensibles, enfermas y tienen miedo de expresar lo que piensan y sienten, por temor a que los profesionales tomen venganza o represalias no enuncian nada, otras manifiestan que si le dan importancia a la falta de respeto, se van a estresar. En la mayoría de los casos, la paciente ubica al médico y a la enfermera en un plano de superioridad debido a sus conocimientos, experiencia y papel de prestador de ayuda, colocándose en un plano de inferioridad, lo que determina, en ocasiones, dificultades en la relación y la desconfianza; asumiendo una postura pasiva, de aceptación a todo lo que dice o le pide el médico, sin que siempre este de acuerdo con él.

Estas situaciones ocasionan tristeza y el llanto, tal como mencionan en los siguientes discursos:

Empecé a llorar, porque, nunca nadie me había visto solamente mi pareja y mi mama cuando me bañaba. Yo me siento muy mal porque debe ser más íntimo más cerrado, solamente el personal que le pertenece debería estar allí, deben proteger al paciente, no exponerlo tanto. MG2

Me siento un poco mal y triste, porque no me gusta que vean mi cuerpo. MG4

El llorar es un mecanismo "reparador psicobiológico"; sin embargo, seguimos teniendo la facultad del Ilanto para liberarnos de la tristeza, tensión emocional, frustración, o incluso emoción. En este sentido la mujer expresa estos sentimientos, cuando se expone a situaciones de desposesión de su intimidad, específicamente de su cuerpo, pues consideran que es una parte de su intimidad muy reservada y no debe quedar a la vista de extraños.

4.2. Reaccionando con vergüenza e incomodidad, con deseo de retiro voluntario.

La vergüenza es ese sentimiento escurridizo cuya sola presencia hace que el individuo se oculte o evite situaciones, personas y sentimientos, buscando un lugar desde el que pasar desapercibido, es también esa convicción, si se quiere irracional, de que el otro nos percibe como seres insuficientes, incompetentes o faltos de belleza 29

Así lo manifiestan las mujeres hospitalizadas:

Da vergüenza estar con harta gente y el doctor que nos examina. Cuando las enfermeras te lavan otros te ven, da vergüenza que vean tu cuerpo, cuando uno está señorita normal, pero cuando ya nos casamos estamos viejas, el cuerpo no es igual, cambia, se mancha, te traen el bacín para orinar, la puerta está abierta, están los 
doctores o los esposos de las pacientes, da vergüenza como mujeres que somos que te vean lo que tienes. MG8

La vergüenza es un sentimiento espontáneo que la persona tiene ante sí misma o ante los demás cuando algo en ella, y por tanto ella misma, aparecen como feos, y por tanto indignos $y$ vituperables ${ }^{29}$. El sentimiento de vergüenza afecta así a lo más íntimo. Yepes ${ }^{1}$ manifiesta que la experiencia interior de la vergüenza es como una enfermedad dentro del yo, una dolencia del alma", un tormento interior o una herida que nos separa de nosotros mismos y de los demás, aislándonos en nuestro sonrojo. En este sentido, el pudor, vergüenza relativa a la desnudez, fue relacionado por los participantes con la invasión de zonas del cuerpo consideradas más íntimas y así lo mencionaron las mujeres hospitalizadas en los anteriores discursos.

Es tan fuerte el efecto emocional que puede causar esta desposesión de su intimidad corporal o psicológica, que muchas veces desean salir de ese ambiente o situación, deseando el retiro voluntario, manifestándolo a sus familiares pero no al personal de salud, pues reconocen su vulnerabilidad y las consecuencias que implicaría esa actuación.

Quería pedir mi alta, pero ahorita no me puedo mover porque tengo que pensar primero en mi hijo, estabilizarme para pedir mi alta, y nunca más volver a pisar acá, prefiero estar en una clínica privada. MG 10

Además la mujer hospitalizada busca la manera de cubrirse, de no exponerse a los demás durante la situación de pérdida de la intimidad, así lo manifiestan:

Mi reacción fue que me cubrí, puse la mano y me cubrí la cara y no le dije nada al médico porque estaba allí rodeada de las alumnas más vergüenza me dio. MG 8

Me cubrí con la sabana, solo eso, que otra reacción voy a tener. $\mathrm{P} 5$
La persona expresa una negativa a que su cuerpo sea tomado, por así decir, sin la persona que lo posee, como una simple cosa, y esto lo confirmamos en las narraciones recogidas, al taparse con las manos o cubrirse con las sabanas.

Allí radica la importancia de pedir su consentimiento cuando se debe tocar su cuerpo, y en protegerlo de miradas de terceros como labor esencial de la enfermera para asegurar el respeto de su intimidad, aunque nuestro entorno no sea es más propicio. Es así que la persona por su naturaleza humana, busca cualquier medida para cubrir su intimidad corporal y no sentirse desprotegida.

\section{Conclusiones}

1. Las enfermeras reconocen la intimidad como un dimensión holística, que implica lo físico, psicológico, espiritual y cultural de la persona, resaltando la corporalidad y la confidencialidad como las esferas más importantes para respetar esta dimensión, sin embargo la mujer hospitalizada percibe la intimidad como el respeto a su cuerpo, relacionado con sus partes íntimas, olvidando los otros ámbitos de la intimidad como dimensión.

2. La intimidad corporal de la mujer fue la más violentada, al afirmar que las exponen frente a miradas extrañas cuando realizan la visita médica, no las cubren con biombos o sábanas durante el aseo genital o la exploración física, y permiten el ingreso de estudiantes sin previo consentimiento y autorización, lo cual genera una contradicción entre los discursos de las enfermeras, pues reconocen que la intimidad debe ser cuidada, incluso enfatizan el aspecto corporal, quizá solo reconocen teóricamente como dimensión holística, pero no la protegen o respetan, por ello las mujeres hospitalizadas exigen y sienten esa necesidad de respeto hacia su cuerpo. 
3. Las enfermeras consideran que la deficiente empatía, capacitación, despersonalización y la resistencia al cambio constituyen condiciones desfavorables para ejercer el cuidado de la intimidad a diferencia de las pacientes que manifiestan que la pérdida de vocación y empatía son situaciones que evitan que se cuide esta dimensión, a su vez ambos sujetos de estudio declaran en sus discursos que estas situaciones se generan por la rutina del profesional y por los años que viene laborando.

4. Las enfermeras y pacientes consideran la infraestructura y los recursos materiales constituyen condiciones desfavorables para ejercer el respeto de la intimidad, la enfermera hace hincapié en que se mejore la infraestructura sin embargo es consciente que estos cambios serán a largo plazo. Además manifiesta que a pesar de esta situación en cuanto a la estructura, depende de la enfermera proteger la intimidad con los medios y materiales que tiene a su alcance. La mujer hospitalizada refiere que, aunque sea la deben cubrir con biombos, también reconoce que es el sistema del hospital que hace aún más que se vulnere la intimidad.

5. Los sentimientos revelados por las pacientes en el contexto hospitalario de este estudio, son predominantemente pasivos, marcados por el silencio, la desatención, la tristeza, y llanto; estos sentimientos se pusieron de manifiesto cuando el personal de salud expuso sus cuerpos frente a miradas extrañas, pues ellas refieren que solo las habían visto desnudas personas muy cercanas como su pareja y mamá, así mismo sintieron vergüenza e incomodidad, estas expresiones contribuyen a una devaluación personal e interfieren en la forma en la que perciben el respeto de esta dimensión. La mujer hospitalizada justifica sus reacciones de pasividad porque manifiesta que está enferma y se siente vulnerable por lo tanto acepta que los profesionales de la salud agredan su intimidad ya que tienen miedo a que estos las traten mal, tomen venganza, renieguen o se desquiten con ellas.

\section{Bibliografía}

1. Yepes R. Fundamentos de Antropología. 6a ed. Pamplona: Eunsa; 1996.

2. Marcuello A. y otros. I Symposium Internacional de Ética en Enfermería. Pamplona: Servicio de Publicaciones, Universidad de Navarra; 1989.

3. Marti M. La intimidad: Conocer y amar la propia riqueza interior. $6 \mathrm{a}$ ed. Madrid: Ediciones Internacionales Universitarias; 2005.

4. Fernández A., Álvarez T., Ramiro J., Martínez S. El respeto a la intimidad. El secreto profesional en Enfermería. Murcia: Asociación Española de Bioética y Ética Médica; 2008.

5. Mozota J., Moliner J., García A., Moreno J., Fernández R., Rabanaqué Percepción de intimidad de los pacientes atendidos en los servicios de urgencias hospitalarios de Aragón. Rev. Emergencias [revista en la Internet] 2013 [acceso 12 de septiembre de 2014]; 25: 445-450. Disponible en: http://www.semes.org/revista_EMERGENCIAS/des cargar/percepcioen-de-intimidad-de-lospacientes-atendidos-en-los-servicios-deurgencias-hospitalarios-de-aragon

6. Ferrer C, Fernández M, Romero MS, Arana JL, Vielva J, Toro R, et al. La intimidad del cuidado y el cuidado de lo íntimo. Reflexiones éticas desde la enfermería. Asociación de bioética fundamental y clínica. Madrid, 2005.

7. Arcos F; Galindo S. La exposición Corporal y el Cuidado de Enfermería. Rev. Cultura de Cuidados [revista en la Internet] 2010 [acceso 12 de septiembre de 2014]; 28(2): 63-71. Disponible en: 
http://scielo.isciii.es/scielo.php?script=sci_arttex t\&pid $=S 113212962008000200006 \& \operatorname{lng}=$ es

8. Ludke M, Marli E.D.A. Pesquisa em Educação: Abordagens Qualitativas. E.P.U. Editora Pedagógica e Universitaria LTDA. André-São Paulo, 1986.

9. Prado M., Souza L., Carraro T. Investigación Cualitativa en Enfermería. EEUU: Organización Panamericana de la Salud; 2008

10. Lupiccino I. Metodología de la Investigación Cualitativa. México: Editorial Limusa; 1997.

11. Colegio de Enfermeros del Perú. Código De Ética Y Deontología. [Sede Web]. Jesús María: enero del 2009 [actualizado el 22 de noviembre de 2005; acceso 17 de noviembre de 2014].disponible en: http://www.cep.org.pe/beta/download/codigo_et ica_deontologia.pdf

12. García F. El derecho a la intimidad del paciente y el secreto profesional de los profesionales de la salud. Rev. Etbio [revista en la Internet]. 2011. [Acceso 27 Mayo del 2015]; 1 (2). Disponible en: http://www.comexbio.org.mx/Documents/2$2011 /$ Garcia\%20Dora.pdf

13. Llaurado G. La autonomía del paciente y la responsabilidad del personal de enfermeria. Rev. De Enfermeria ENE. [Revista en la Internet]. 2013. [Acceso 07 de Octubre del 2015]; 7(1). Disponible en: eneenfermeria.org/ojs/index.php/ENE/article/downl oad/211/pdf

14. Gómez L. El cuidado de enfermería del paciente en estado crítico una perspectiva bioética. Universidad de La Sabana, Chía, Cundinamarca: Colombia; 2008. Disponible en: http://personaybioetica.unisabana.edu.co/index. $\mathrm{php} /$ personaybioetica/article/view/966/1307

15. Bórquez G, Raineri G, Bravo M. La evaluación de la «capacidad de la persona»: en la práctica actual $y$ en el contexto del consentimiento informado. Rev. Médica de Chile [revista en la Internet]. Octubre 2004. [Citado 01 de noviembre del 2015]; 132 (10): 1243-1248 Disponible en: http:/ / www.scielo.cl/scielo.php?script=sci_arttext \&pid $=$ S0034-98872004001000013

16. Díaz $\mathrm{P}$, Ullate $\mathrm{S}$. El consentimiento informado en la práctica asistencial. Universidad de Cantabria: España; 2003. Disponible en: http://repositorio.unican.es/xmlui/bitstream/han dle $/ 10902 / 3015 /$ DiazBurguilloP.pdf? sequence $=1$

17. Yepes R. La elegancia, algo más que buenas maneras. Rev. Nuestro Tiempo [revista en la Internet]. Octubre 1966. [Citado 01 de noviembre del 2015]; 508: 110-123 Disponible en: http://www.encuentra.com/revista/wpcontent/uploads/2012/07/elegancia_la_elegancia _algo_mas_que_buenas_maneras.pdf

18. Ruiz F. Intimidad y confidencialidad en la relación clínica. Rev. Persona y Derecho [revista en la Internet]. Setiembre 2013. [Citado 01 de noviembre del 2015]; 69: 53-101 Disponible en: www.unav.edu/publicaciones/revistas/index.php/ persona-y.../1443

19. Quinzano E. La enfermería ante el secreto profesional. Escuela Universitaria de Enfermería" Casa de salud Valdecilla" Departamento de enfermería- universidad de Cantabria: España; 2012/2013 Disponible en: http://repositorio.unican.es/xmlui/bitstream/han dle/10902/2977/JordanQuinzanoE.pdf?sequence $=1$

20. Burrows J, Echeverría C, Goic A, Herrera C, Quintana C, Rojas A. El respeto a la intimidad del paciente. Rev. méd. Chile [revista en la Internet]. 2014 Abr [Acceso 2014 Oct 29]; 142(4): 506-511. Disponible en: http: / / www.scielo.cl/scielo.php?script=sci_arttext \&pid=S0034-98872014000400013\&lng=es.

http://dx.doi.org/10.4067/S0034$\underline{98872014000400013}$ 
21. González L, Velandia A., Flores V. Humanización del cuidado de enfermería. Rev. CONAMED, Suplemento de enfermería [revista en internet] 2009 [citado 1 de noviembre de 2015]; 14(1): 40-43. Disponible en: https://dialnet.unirioja.es/descarga/articulo/363 3444.pdf

22. Bernal A. La capacitación del personal de enfermería. Su repercusión en la calidad de los servicios. Rev. Electrónica de las ciencias médicas en Cienfuegos- Medisur [revista en internet] 2011 mayo [acceso 5 de noviembre de 2015]; 9(3): 89. Disponible en: file:///C:/Documents\%20and\%20Settings/alfonso /Mis\%20documentos/Downloads/La\%20capacitaci \%C3\%B3n\%20del\%20personal\%20de\%20enfermer\% C3\%ADa.\%20Su\%20repercusi\%C3\%B3n\%20en\%20la \%20calidad\%20de\%20los\%20servicios.pdf

23. Guevara B. Una aproximación al perfil de la enfermera intensivista. Enfermería Cuidados Intensivos y Cuidados Críticos. 10/23/2007. Revista Electrónica Portales Médicos.com. Disponible

en:http://www.portalesmedicos.com/publicacion es/articles/756/ 1 /Una-aproximacion-al-perfilde-la-enfermera-intensivista.html[Consultado el 3.10.2014].

24.Rodríguez A. Enfermería ante la deshumanización: una necesidad por ser considerada desde la filosofía de Emmanuel Levinas. Rev. Enfermería en Costa Rica [revista en internet] julio 2011 [acceso 10 de noviembre de 2015]; 32(1): 37-42. Disponible en: http://www.binasss.sa.cr/revistas/enfermeria/v3 $\underline{2 n 1 / a r t 7 . p d f}$

25. Rubín M. [sede Web].España: Lifeder.com; 2001 [actualizado el 10 de octubre del 2014; acceso 29 de octubre del 2015]. Resistencia al cambio: causas y cómo manejarlo; Disponible en: http://www.lifeder.com/resistencia-al-cambio/

26. BUAPAS123456. [Sede Web].España: Lifeder.com; 2001 [actualizado el 10 de mayo del 2014; acceso 30 de octubre del 2015]. LA ETICA Y LA MORAL DE LA ENFERMERIA; Disponible en: http://buapas123456.wikispaces.com/

27. Gonzales J, Quinteros M. Percepción del cuidado humanizado en pacientes que ingresan al servicio de urgencias de ginecoobstetricia de una institución de II y III nivel de atención. [Tesis para licenciatura].Bogotá D.C: Facultad de enfermería, Pontifica universidad Javeriana; 2009.

28. Burgos M, Paravic K. Percepción de violencia de los pacientes hospitalizados en los servicios de medicina y cirugia de un hospital público. Cienc. enferm. [Internet]. 2003 Dic [citado 2015 Nov 01]; 9(2): 29-42. Disponible en: http:/ / www.scielo.cl/scielo.php?script=sci_arttext \&pid $=$ S071 7-95532003000200004\&lng=es. http://dx.doi.org/10.4067/S071795532003000200004

29. Gomes de Amorim A, Kay Nations M, Costa M. Sentimientos, sentidos y significados en pacientes de hospitales públicos en el noreste de Brasil. Rev. Salud pública [revista en internet] setiembre 2009 [acceso 10 de noviembre de 2015]; 11(5): 754-765. Disponible en: http://www.scielosp.org/pdf/rsap/v1 ln5/v1 ln5a 08.pdf 